\title{
Clinical experiences in Turkish paediatric patients with chronic recurrent multifocal osteomyelitis
}

\author{
Betül Sözeri' ${ }^{1}$, Nuray Aktay Ayaz², Başak Yıldız Atıkan, Şerife Gül Karadağ², \\ Mustafa Çakan², Mehmet Argın", Murat Sezak ${ }^{5}$ \\ ${ }^{1}$ Division Pediatric Rheumatology, Health Sciences University, Istanbul Umraniye Training and Research Hospital; \\ ${ }^{2}$ Division Pediatric Rheumatology, Health Sciences University, Istanbul Kanuni Sultan Suleyman Education and Research \\ Hospital; Divisions ${ }^{3}$ Pediatric Infectious Diseases, ${ }^{4}$ Radiology and ${ }^{5}$ Pathology, Ege University Faculty of Medicine, Izmir, \\ Turkey.E-mail: betulsozeri@yahoo.com \\ Received: 5th June 2017, Revised: 9th September 2017, 15th October 2018, Accepted: 23rd October 2018
}

SUMMARY: Sözeri B, Aktay Ayaz N, Yıldız Atıkan B, Karadağ ŞG, Çakan M, Argın M, Sezak M. Clinical experiences in Turkish paediatric patients with chronic recurrent multifocal osteomyelitis. Turk J Pediatr 2019; 61: 879-884.

Chronic recurrent multifocal osteomyelitis (CRMO) is a clinical entity which occurs mainly in children and adolescents with recurrent episodes of pain occurring over several years. Cause and physiopathology of disease is still uncertain. We aim to assess clinical characteristics and treatment options, need and response to anti-inflammatory therapies in children diagnosed chronic recurrent multifocal osteomyelitis

Demographic data and clinical features of seventeen children diagnosed with CRMO in 2 pediatric rheumatology centers in Turkey were reviewed retrospectively. The diagnosis was based on clinical findings, radiological images and histopathological and microbiological studies.

A total of 17 patients were included in the study. The median age of diagnosis was 9.6 44.2 years. The mean follow-up time was 31.6 months (range 6-35 months). Most patients ( $\mathrm{n}: 10)$ had a recurrent multifocal disease course (>6 months), 6 patients had a persistent course and a patient had only one episode of CRMO. MEFV gene mutations were detected in 4 patients whose clinical features reduced with colchicine therapy. All patients had received nonsteroidal anti-inflammatory drugs but only one had complete response. Thirteen children with NSAID failure subsequently received corticosteroids, sulfasalazine, methotrexate, Anti TNF $\alpha$ drugs, or a combination of these drugs.

This study is the largest cohort of pediatric CRMO patients in our country. Clinical evolution and imaging investigations should be closely done to avoid delays in diagnosis. Ethnic differences create changes in the presentation of the disease and response to treatment

Key words: chronic recurrent multifocal osteomyelitis, diagnosis, therapy.

Chronic recurrent multifocal osteomyelitis (CRMO) is characterized by a prolonged, fluctuating course with recurrent episodes of pain. Giedion et al. ${ }^{1}$ first described the condition in 1972 as an unusual form of multifocal bone lesions with subacute and chronic symmetrical osteomyelitis. Unifocal and non-recurrent patterns also have been recognized. ${ }^{2}$ In typical cases, multiple bone lesions with apparent bone destruction, osteolysis, sclerosis, and hyperostosis are seen. ${ }^{3}$ But there are variable clinical manifestations, which make differential diagnosis of CRMO often difficult. The diagnosis of this entity is based on the following criteria: bone lesions with a radiographic picture suggesting subacute or chronic osteomyelitis; an unusual location of lesions when compared with infectious osteomyelitis 
with a frequent involvement of the clavicle and frequently multifocal; no abscess formation, fistula or sequestrate; lack of a causative organism; nonspecific histopathological and laboratory findings compatible with subacute or chronic osteomyelitis; a characteristic prolonged, fluctuating course with recurrent episodes of pain occurring over several years; and occasional accompanying skin diseases. ${ }^{4,5}$ Bone lesions can occur at any site of the skeleton except for the neurocranium. ${ }^{6}$ Exact molecular pathophysiology of CRMO remains incompletely understood. Recent findings indicate that an imbalance between pro- (IL-6, TNF- $\alpha$ ) and anti-inflammatory (IL-10) cytokines may be centrally involved in the molecular pathology of CRMO. ${ }^{6}$ Sterile bone inflammation is a hallmark of CRMO and other autoinflammatory bone disorders which are listed as PAPA (pyogenic arthritis, pyoderma gangrenosum and acne syndrome), DIRA (deficiency of IL-1 receptor antagonist), familial chronic multifocal osteomyelitis which is also referred to as Majeed syndrome and SAPHO (synovitis, acne, pustulosis, hyperostosis and osteitis) syndrome.

The diagnosis is based on the exclusion of infective and malign causes. Hematogenous spread of an infection seems unlikely because pathogens are generally not cultured from the blood, bones or joints of the patients. But there is strong evidence for inflammation and this clinical entity is thought to be in the spectrum of autoimmune and autoinflammatory disorders. ${ }^{7}$ The presence of higher rates of systemic inflammatory conditions, particularly psoriasis and inflammatory bowel disease (IBD) in patients and family members also supports the idea. ${ }^{8}$ Kaiser et al. ${ }^{9}$ concluded that an osteomyelitis in the pelvic region is significantly associated with other features of juvenile spondyloarthritis in the large group.

In this study we aimed to assess clinical characteristics and treatment options, need and response to anti-inflammatory therapies in children.

\section{Material and Methods}

Demographic data and clinical features of seventeen children diagnosed with CRMO in two pediatric rheumatology centers in
Turkey were reviewed retrospectively. The diagnosis was based on clinical findings, radiological images and histopathological and microbiological studies. Age, gender, disease duration, locations of lesions in X-rays, and magnetic resonance imaging (MRI) findings of histopathological and microbiological studies if conducted, laboratory investigations at diagnosis (acute phase reactants and whole blood count), treatment modalities, number of flares and MEFV mutation in selected cases were noted. SPSS 16.0 was used to analyses the descriptive statistics.

The study was approved by the Local Ethics Committee. The informed consent was signed by all patients and or their parents. All authors have contributed substantially to, and are in agreement with the content of the manuscript. The authors have no conflicts of interest.

\section{Results}

Seventeen patients were assessed ( 8 females and 9 males) with a median age of $9.6 \pm 4.2$ years (range 4-17) at diagnosis over two years. Median duration since diagnosis was 1.4 years and median duration of active disease was 1.1 years. The mean follow-up time was 31.6 months (range 6-35 months). Patient's characteristics are summarized in Table I. Five patients were referred from pediatricians, three from pediatric infectious disease specialist, four from orthopedics and five from general practitioner. The most common symptom was localized pain and swelling and the patients were pre diagnosed with acute osteomyelitis (n: 9), fibroma (n: 1), familial Mediterranean fever (n: 2) and sarcoma (n: 4).

Over the course of the disease, 90 lesions were identified, with a median of 4 lesions per child (Table II). Ten of the patients had recurrent multifocal lesions, 6 had persistent multifocal lesions while only one patient had unifocal non recurrent lesion during the course of the disease. The most common sites for bony involvement were the tibia (11) femur (8) and vertebrae (6) followed by metatarsal bones (4), iliac bone (4), scapula (3), clavicle (2), talus (2), calcaneus (2) and the humerus (1). In addition, six patients had synovitis at one or more joints that were adjacent to bony lesions. 
Table I. Demographic, Clinical and Radiological Characteristics of Patients with Chronic Recurrent Multifocal Osteomyelitis (n: 17).

\begin{tabular}{lc}
\hline Characteristics & Results \\
\hline Female, n (\%) & $8(47.1 \%)$ \\
Mean age at diagnosis, years & $9.6 \pm 4.2$ \\
$\begin{array}{l}\text { Median duration since diagnosis } \\
\text { (from symptom onset), years }\end{array}$ & 1.4 \\
Median duration of active disease, & 1.1 \\
years & \\
Median number of lesions per patient & 4 \\
Median number of flare per patient & 2.6 \\
Accompanied clinical signs, n (\%) & \\
$\quad$ Arthralgia & $10(58.8 \%)$ \\
$\quad$ Arthritis & $3(17.6 \%)$ \\
$\quad$ Fever & $2(11.8 \%)$ \\
$\quad$ Pustulosis & $1(5.9 \%)$ \\
HLA B27 positivity, n (\%) & $2(11.8 \%)$ \\
Family history, n $(\%)$ & $2(11.8 \%)$ \\
\hline
\end{tabular}

Table II. Distribution of 90 Lesions in Patients.

\begin{tabular}{lc}
\hline Localization & $\begin{array}{c}\text { Results, } \mathrm{n} \\
(\%)\end{array}$ \\
\hline Lower limb lesions & $32(35.6)$ \\
Axial skeleton (vertebrae, iliac bone) & $28(31.1)$ \\
Chest wall & $7(7.8)$ \\
Other & $23(25.6)$ \\
Talus, navicular, calcaneus & $8(8.9)$ \\
Metatarsal bones & $13(14.4)$ \\
Humerus & $1(1.1)$ \\
Metacarpal bones & $1(1.1)$ \\
\hline
\end{tabular}

Complete blood count was normal in 12 patients, two had mild anemia. Nine patients had elevated erythrocyte sedimentation rate (ESR (maximum $96 \mathrm{~mm} /$ hour) and C-reactive protein (CRP). Patients with family history with rheumatic diseases or elevated inflammatory markers were tested for HLA-B27 and two were found to be positive. All patients were first examined with a plain X-ray but further tests with CT scans and MRI's were necessary and they showed bone marrow edema, heterogeneous sclerosis, periost reaction (Fig. 1).
Bone biopsy was performed in 9 patients. Nonspecific chronic osteomyelitis accompanied with both lymphocytic and macrophage infiltration and fibro-sclerosis were detected in seven of them. Two samples were found to have neutrophils infiltration predominating. All microbiological cultures were negative for any specific infectious agent.

Nine of these ten patients were treated with antibiotics at the time of the initial diagnosis where acute bacterial osteomyelitis was suspected. Nonsteroidal anti-inflammatory drugs (NSAIDs) were used in all of the patients initially, four patients had complete response. Oral corticosteroids were used in seven patients but additional therapy [sulfasalazine (SSZ) and/or methotrexate (MTX)] was necessary in 11 patients for steroid dependency. By SSZ, MTX and anti TNF- $\alpha$ (etanercept) remission was achieved in $60 \%$ of treated patients (6 of 10), $33 \%$ (2 of 6 ) and $75 \%$ (3 of 4 ), respectively. At the last visit, disease was considered to be in remission in 13 of 17 patients, of whom $52.9 \%$ (n: 9) were

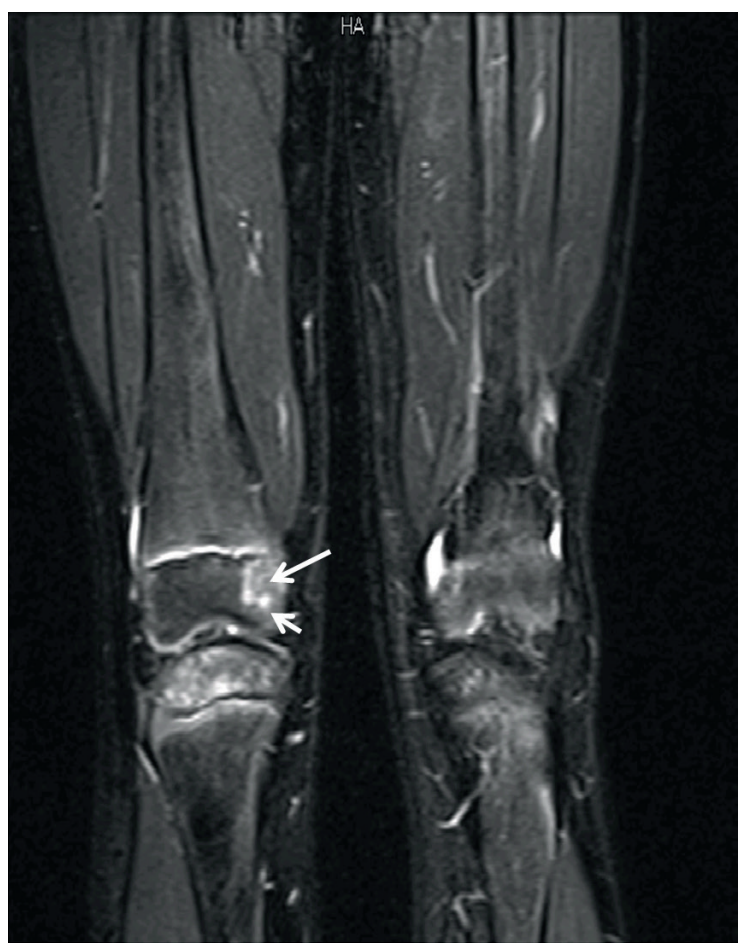

Fig. 1. Knee section of T2-short time inversion recovery (STIR) whole-body magnetic resonance image of a patient with chronic recurrent multifocal osteomyelitis. The arrow shows hyperintensity of bone. 
still receiving therapy. Two patients who were carrying HLA B27 antigen with active disease received anti TNF- $\alpha$ therapy and NSAID therapy only during painful episodes at the last visit.

\section{Discussion}

In this study we presented our experience in CRMO and showed similarities to other pediatric series. CRMO was first described in European Caucasians and most reports are from Europe and North America. ${ }^{6}$ Girschick et al. $^{10}$ recently published the largest case series of $\mathrm{CNO}$ patients; they reported 64 percent of patients were female and disease onset at a mean age of 9.9 years. Conversely, our cohort had male gender dominance (9:8). We think that this variability is due to our racial characteristics, and it has been found that Turkish patients were not taken into their cohort. The mean age of disease onset $(9.6 \pm 4.2$ years $)$ and also delay in diagnosis which is supported with a mean duration of active disease without effective treatment (13 months) were similar to other pediatric groups. ${ }^{5,8-12}$ Correct diagnosis of CRMO is often delayed because the clinical symptoms are unspecific such as, pain local swelling, redness skin rashes.

Initial symptoms were reported as bone pain with or without local swelling and warmth, malaise, mild fevers and even fractures in previous studies. ${ }^{9-12}$ There was bone pain in all of our patients like other patients at the onset of disease. Painful lesions from a total of 90, thirty-two have been identified in the lower limbs (35.5\%). Axial involvement (iliac bone and vertebra) were similar $(31.1 \%)$ to the previously described. ${ }^{8,11,12}$ Two of our patients had single initial lesion at the time of diagnosis whereas the other fifteen had multiple lesions. The number of flares per patient differed between 1 and 9 .

There are some organisms like Mycoplasma hominis and Proprionibacterium acnes suspected as the trigger of the disease ${ }^{2,12}$ but we did not find any clue of infection or any association between infection and recurrent osteomyelitis.

The acute phase response is usually determined as high in CRMO (50-90\% of cases) at the time of diagnosis., ${ }^{5-8-12}$ In our group, 10 patients (59\%) had elevated CRP and/or ESR levels similarly.

Mixed osteolytic and sclerotic lesions in especially the metaphysis of the long bones, are one of the most common radiologic findings in the CRMO patients. ${ }^{13,14}$ Very early in the disease plain films may be normal or only show osteopenia; however, increased uptake on technetium bone scan or evidence of marrow edema on MRI can be seen at this stage. As consistent with previous studies ${ }^{12,15}$, we have also detected more lesions with MRI than bone scintigraphy.

CRMO is an autoinflammatory disorder of innate immunity because the disease is characterized by episodes of systemic inflammation occurring in the absence of autoantibodies. ${ }^{15}$ The familial Mediterranean fever (FMF) is a prototype of autoinflammatory diseases and quite common in our country and it is also tends to be related with the inflammatory process in CRMO. Shimizu et al. $^{17}$ also described a case of colchicineresponsive CRMO with Mediterranean fever (MEFV) gene mutations and implied that the MEFV gene might be associated with CRMO. Therefore we performed mutation analysis in the MEFV gene and heterozygote mutations were detected in 4 patients. We observed reduced severity of clinical features with colchicine therapy in these patients. We suggest that MEFV gene might be associated with CRMO and colchicine therapy should be discussed as a possible treatment alternative for the disease. But a large number of patients are needed to be sure.

The alternative hypothesis is that CRMO belongs to the group of spondyloarthritides due to the low frequency of HLA-B27 $(7 \%) .{ }^{12}$ In our patients, 2 had HLAB-27. It was remarkable that patients with HLA-B27 mutations needed further investigations and treatment with anti-TNF agents. It can be hypothesized that genetic and/or ethnic differences can affect the course of the disease. But further studies are necessary to support the possible relationship between ethnicity and specific genetic mutations.

NSAIDs are used as a first line treatment 
strategy in CRMO, which provided pain reduction and achieved remission in most CRMO patients. ${ }^{18,19}$ In our cohort, NSAIDs were prescribed to all patients, but adequate control of the disease was achieved in four cases. Other agents including corticosteroids, sulfasalazine, methotrexate or cytokinedirected therapies were reported as secondline treatment. Corticosteroids have been applied in short-term high-dose regimens or continuously in lower doses. ${ }^{2}$ Sulfasalazine and methotrexate have been reported as effective and safe in NSAID-refractory patients. ${ }^{9,12}$ Anti TNF- $\alpha$ drugs are the most frequently used biologic agents. Borzutzky et al. ${ }^{7}$ reported that $20 \%$ had clinical remission treated with methotrexate while $46 \%$ had clinical remission with TNF- $\alpha$ inhibitors in 70 patients with CRMO. Similar results have been reported in subsequent studies., ${ }^{9}, 12,20-22$ In addition, the bisphosphonate therapy was found to be effective in CRMO especially at vertebral involvement. ${ }^{22}$ In our cohort, oral corticosteroids were used as a bridging therapy for a short time before sulfasalazine and/or methotrexate. They were necessary in 10 patients for steroid dependency and were successful in approximately $20-40 \%$ of these patients. We have observed that anti TNF -alpha therapy provided remission in treatment-resistant cases similar to previous studies.

The clinical course of CRMO is variable, in a German cohort of 89 patients $20 \%$ of patients had bone inflammation in one location without recurrence, nearly $45 \%$ of patients had classic CRMO with multiple bone lesions with recurrent flares with remissions, and the remaining $35 \%$ had persistent multifocal bone inflammation for longer than 6 months without remissions (persistent multifocal). ${ }^{8}$ In our cohort, 90 lesions were identified. Ten patients $(59 \%)$ had recurrent multifocal lesions, $6(35.3 \%)$ had persistent multifocal lesions while only one patient $(5,7 \%)$ had unifocal non recurrent lesion during the course of the disease. We also evaluated all the patients with the Ped CNO score suggested by Beck et al. ${ }^{24}$ at the last visit, 4 patients $(23.5 \%)$ reached Ped CNO 70 while 11 patients $(64.7 \%)$ reached Ped CNO 50. The remaining 2 patients had active disease.
This study is the largest Turkish cohort of pediatric CRMO patients. Our study has limitations since the cohort is small and the study had been conducted retrospectively. It is not possible to declare precise conclusions about the cause or best treatment modalities. This report indicates that CRMO may be overlooked and misdiagnosed at first sight in our community. Although it is known as a benign disease, recurrences and subclinical attacks with mild pain in extremities without any significant increase in inflammatory markers are frequently seen. Therefore it is necessary to follow up these patients closely to avoid potential complications and sequela.

\section{REFERENCES}

1. Giedion A, Holthusen W, Masel LF, Vischer D. Subacute and chronic "symmetrical" osteomyelitis. Ann Radiol 1972; 15: 329-342.

2. Girschick HJ, Raab P, Surbaum S, et al. Chronic nonbacterial osteomyelitis in children. Ann Rheum Dis 2005; 64: 279-285.

3. El-Shanti HI, Ferguson PJ. Chronic recurrent multifocal osteomyelitis: a concise review and genetic update. Clin Orthop Relat Res 2007; 462: 11-19.

4. Bjorksten B, Gustavson KH, Eriksson B, Lindholm A, Nordström S. Chronic recurrent multifocal osteomyelitis and pustulosis palmoplantaris. J Pediatr 1978; 93: 227-231.

5. Beretta-Piccoli BC, Sauvain MJ, Gal I, et al. Synovitis, acne, pustulosis, hyperostosis, osteitis (SAPHO) syndrome in childhood: a report of ten cases and review of the literature. Eur J Pediatr 2000; 159: 594601.

6. Hedrich CM, Hofmann SR, Pablik J, Morbach $\mathrm{H}$ Girschick HJ. Autoinflammatory bone disorders with special focus on chronic recurrent multifocal osteomyelitis (CRMO). Pediatr Rheumatol Online J 2013; 11: 47.

7. Borzutzky A, Stern S, Reiff A, et al. Pediatric chronic nonbacterial osteomyelitis. Pediatrics 2012; 130: 1190-1197.

8. Jansson A, Renner ED, Ramser J, et al. Classification of non-bacterial osteitis: retrospective study of clinical, immunological and genetic aspects in 89 patients. Rheumatology (Oxford) 2007; 46: 154-160.

9. Kaiser D, Bolt I, Hofer M, et al. Chronic nonbacterial osteomyelitis in children: a retrospective multicenter study. Pediatr Rheumatol Online J 2015; 13: 25. 
10. Girschick H, Finetti M, Orlando F, et al. The multifaceted presentation of chronic recurrent multifocal osteomyelitis: a series of 486 cases from the Eurofever international registry. Rheumatology (Oxford) 2018; 57: 1504.

11. Walsh P, Manners PJ, Vercoe J, Burgner D, Murray KJ. Chronic recurrent multifocal osteomyelitis in children: nine years' experience at a statewide tertiary paediatric rheumatology referral centre. Rheumatology (Oxford) 2015; 54: 1688-1691.

12. Wipff J, Costantino F, Lemelle I, et al. A large national cohort of French patients with chronic recurrent multifocal osteitis. Arthritis Rheumatol 2015; 67: $1128-1137$

13. Khanna G, Sato TS, Ferguson P. Imaging of chronic recurrent multifocal osteomyelitis. Radiographics 2009; 29: 1159-1177.

14. Wipff J, Adamsbaum C, Kahan A, Job-Deslandre C. Chronic recurrent multifocal osteomyelitis. Joint Bone Spine 2011; 78: 555-560.

15. Morbach H, Schneider P, Schwarz T, et al. Comparison of magnetic resonance imaging and $99 \mathrm{~m}$ Technetiumlabelled methylene diphosphonate bone scintigraphy in the initial assessment of chronic non-bacterial osteomyelitis of childhood and adolescents. Clin Exp Rheumatol 2012; 30: 578-582.

16. Roderick MR, Ramanan AV. Chronic recurrent multifocal osteomyelitis. Adv Exp Med Biol 2013; 764: 99-107.

17. Shimizu M, Tone $\mathrm{Y}$, Toga A, et al. Colchicineresponsive chronic recurrent multifocal osteomyelitis with MEFV mutations: a variant of familial Mediterranean fever? Rheumatology (Oxford) 2010; 49: 2221-2223.
18. Catalano-Pons C, Comte A, Wipff J, et al. Clinical outcome in children with chronic recurrent multifocal osteomyelitis. Rheumatology 2008; 47: 1397-1399.

19. Falip C, Alison M, Boutry N, et al. Chronic recurrent multifocal osteomyelitis (CRMO): a longitudinal case series review. Pediatr Radiol 2013; 43: 355-375.

20. Deutschmann A, Mache CJ, Bodo K, Zebedin D, Ring E. Successful treatment of chronic recurrent multifocal osteomyelitis with tumor necrosis factoralpha blockage. Pediatrics 2005; 116: 1231-1233.

21. Marangoni R, Aris S, Halpern R. Chronic recurrent multifocal osteomyelitis primarily affecting the spine treated with anti-TNF therapy. Spine (Phila Pa 1976) 2010; 35: 253-256

22. Batu ED, Ergen FB, Gulhan B, Topaloglu R, Aydingoz $\mathrm{U}$, Ozen S. Etanercept treatment in five cases of refractory chronic recurrent multifocal osteomyelitis (CRMO). Joint Bone Spine 2015; 82: 471-473.

23. Hospach $\mathrm{T}$, Langendoerfer $\mathrm{M}$, von Kalle $\mathrm{T}$, Maier J, Dannecker GE. Spinal involvement in chronic recurrent multifocal osteomyelitis (CRMO) in childhood and effect of pamidronate. Eur J Pediatr 2010; 169: 1105-1111.

24. Beck C, Morbach $\mathrm{H}$, Beer $\mathrm{M}$, et al. Chronic nonbacterial osteomyelitis in childhood: prospective follow-up during the first year of anti-inflammatory treatment. Arthritis Res Ther 2010; 12: 74. 